\title{
HLA-DR Mismatch Count
}

National Cancer Institute

\section{Source}

National Cancer Institute. HLA-DR Mismatch Count. NCI Thesaurus. Code C128963.

The determination of the number of mismatch between the study subject and his or her donor for the human leukocyte antigen, class II, antigen-D-related (HLA-DR). 